\title{
Optical properties and catalytic activity of bimetallic gold-silver nanoparticles
}

Lili Feng*, Guo Gao, Peng Huang, Kan Wang, Xiansong Wang, Teng Luo, Chunlei Zhang

Key Laboratory for Thin Film and Microfabrication Technology of Ministry of Education, National Key Laboratory of Micro/Nano Fabrication Technology,Research Institute of Micro/Nano Science and Technology, Shanghai Jiao Tong University, Shanghai,200240, P. R. China

*Corresponding author: 1lfeng@sjtu.edu.cn

\begin{abstract}
Gold-silver bimetallic nanoparticles (including alloy, core-shell and nanocage structure) are good noble metal nanomaterials with unique properties which have widespread applications in electronic, photonic, chemical and biological fields. Especially in recent years, more and more investigators are motivating this advanced material rapidly towards surface-enhanced raman scattering (SERS) and high catalytic activity of carbon monoxide oxidation at room temperature. Herein, we outlined the current research advances of gold-silver bimetallic nanoparticles in synthesis, optical properties, surfaceenhanced raman scattering application and catalytic activity, with the aim of stimulating more research to achieve more useful application as soon as possible.
\end{abstract}

Key Words: gold-silver alloy nanoparticles, gold-silver core-shell nanoparticle, nanocage, surface plasmon resonance, surface-enhanced raman scattering, catalysis

Citation: L. Feng, et al. Optical properties and catalytic activity of bimetallic gold-silver nanoparticles. Nano Biomed. Eng. 2010, 2(4), 258-267. DOI: $10.5101 /$ nbe.v2i4.p258-267.

\section{Introduction}

In recent years, investigation of nanostructured materials, especially metal nanoparticles, has attracted much attention because of their interesting chemical, mechanical, and physical properties, and potential applications in nanoelectronic and optoelectronic devices [1-14]. The optical properties of these nanomaterials are due to the surface plasmon resonance, which is a collective oscillation of conduction electrons of noble metals. The optical properties of gold, silver, and their bimetallic nanoparticles change with size, shape, and dielectrics of the medium [15-25]. Nowadays, much effort has been devoted to the fabrication of metal nanocomposites, including alloys [26-29], core-shell and mixed particles [30-37], owing to their valuable non-linear optical properties in optical switches [38,39], in catalysis [40,41], and in surface-enhanced raman scattering(SERS) [42,43], due to new bifunctional or synergistic effects.

Gold and silver colloids display different optical properties. Whereas Ag colloids display an absorption maximum at about $420 \mathrm{~nm}$, the Au colloids show a maximum at about $550 \mathrm{~nm}$. When gold-silver alloy nanoparticles were prepared, the plasmon maximum is between the absorption maximum of Ag colloids and Au colloids. The plasmon maximum of gold-silver alloy nanoparticles has a blue-shifted with an increasing amount of silver, and a linear relationship is found [44-46]. When $\mathrm{Au} / \mathrm{Ag}$ core/shell nanostructure was synthesized, there are two absorption bands in the UV-Vis spectrum $[47,48]$. The difference in absorption bands is able to differentiate gold-silver bimetallic structure. Moreover, the plasmon bandwidth (fwhm) is sensitive to the fraction of silver in alloy [19]. The plasmon band becomes sharper and also increases in intensity as the fraction of silver increases. A narrower bandwidth allows much easier detection of the change in the surface plasmon band maximum in response to refractive index changes in the surrounding medium. Ag nanoparticles are predicted to be more sensitive in detecting smaller amounts of analyte, by the measurement of the shift in the position of the plasmon bands as well as changes in the band intensity. In addition, silver and gold colloids are most frequently employed metallic systems 
in SERS. In comparison with silver, gold surface shows a lower surface enhancement factor (EF) in the visible region. So many attempts to obtain silver/gold composite particles have been carried out in order to induce a greater SERS enhancement on gold surface.

Apart from the optical properties, Ag, Au, and their bimetallic nanoparticles have excellent catalytic activity. Haruta and co-workers discovered the catalysis of gold nanoparticles in a class of oxygen-transfer reactions near ambient temperature [49]. In particular, the oxidation of carbon monoxide has attracted a lot of interest. It is expected to prevent catalyst poisoning by carbon monoxide in methanol fuel cells, and save people from carbon monoxide poisoning in fire. While gold nanoparticles adsorb CO molecules well, they do not strongly adsorb and activate oxygen molecules [5052]. Many reference reported $\mathrm{Au}-\mathrm{Ag}$ alloy nanoparticles strongly enhanced oxidation of carbon monoxide [11,5355]. The catalytic activity has been found to be sensitive to the size of the nanoparticles, Ag contents, the nature of the support, and the preparation methods. Therefore, most of the reported works focused on tuning of the particle size, modification of the support, and the pretreatment conditions.

Herein, we outlined the current research advances of gold-silver bimetallic nanoparticles in synthesis, optical properties, surface-enhanced raman scattering and catalytic activity, with an aim of stimulating more research to achieve practical application as soon as possible.

\section{Synthesis and Optical properties}

In material science, the range of properties of metallic systems can be greatly extended by taking mixtures of elements to generate intermetallic compounds and alloys. In many cases, there is an enhancement in specific properties upon alloying due to synergistic effects. The rich diversity of compositions, structures, and properties of metallic alloys has led to widespread applications in electronics, engineering, and catalysis. The structures of bimetallic have crucial effect on the properties. Generally speaking, nanoalloys can be classified to three architectures according to their mixing pattern and geometric structure [16]. They are core-shell, subcluster segregated, and mixed architectures (The structure was shown in Figure 1). Core-shell nanostructures consist of a shell of one type of atom surrounding a core of another, though there may be some mixing between the shells. This structure is of the most common to a large variety of systems. For instance, there are many reports on preparing AucoreAgshell (AgcoreAushell) nanoparticles and nanorods [30,31,43,56-61]. Mixed nanoalloys may be either ordered or random, and the random mixing pattern is common to many systems. In bulk, gold and silver are miscible, and many reports have been involved with goldsilver alloy [27,44,45,62-67]. But some investigators reported gradient alloy formation for gold rich in the core and silver rich in the shell in nanometer scale, although it is still called as gold-silver alloy [62,68]. Apart from coreshell and mixed architectures, nanocage structure for gold and silver also attracts great intention for their biosensor, photothermal therapy and catalytic application [28, 6973].

To make different alloy structures, different synthetic methods are required. The most common method is coreduction of gold and silver precursor by strong reducing agent. For example, their preparation has been achieved by citrate reduction $[44,74]$, borohydride reduction $[45,75]$, hydrazine hydrate and hydroxylamine reduction [76]. Apart from different reducing agent, different processes are studied too, such as electrochemical reduction, photolytic reduction, radiolytic reduction, water-in-oil microemulsions processing [67,77-79], laser ablation [47,63,80,81], solvent extraction-reduction, and evaporation-condensation. M. A. El-Sayed and coworkers prepared gold-silver alloy nanoparticles with varying mole fractions by the co-reduction of chlorauric acid $\left(\mathrm{HAuCl}_{4}\right)$ and silver nitrate $\left(\mathrm{AgNO}_{3}\right)$ with sodium citrate [44]. As the optical absorption spectra of their solutions shows only one plasmon absorption, it is concluded that co-reduction of chlorauric acid $\left(\mathrm{HAuCl}_{4}\right)$ and silver nitrate $\left(\mathrm{AgNO}_{3}\right)$ with sodium citrate leads to a homogeneous formation of alloy nanoparticles. The plasmon maximum blue-shifts linearly with increasing silver content. UV-vis absorption spectra of gold and gold-silver alloy nanoparticles with varying gold mole fractions was shown in Figure 2. Compared to alloy nanoparticles, Luis M. Liz-Marzàn research group proved there were two maximum of the plasmon band for coreshell structure [76]. UV-vis absorption spectra of gold and gold-silver core-shell nanoparticles was shown in Figure 3. Catherine J. Murphy and coworkers synthesized gold-silver alloy nanoparticles with reducing $\mathrm{HAuCl}_{4}$ and $\mathrm{AgNO}_{3}$ by sodium borohydride in the presence of sodium citrate as a capping agent, in water [45]. The problem in co-reduction of gold and silver precursor by strong reducing agent is precursor concentrations in solution. The concentration needs dilute enough to avoid $\mathrm{AgCl}$ precipitation from $\mathrm{HAuCl}_{4}$ and $\mathrm{AgNO}_{3}$
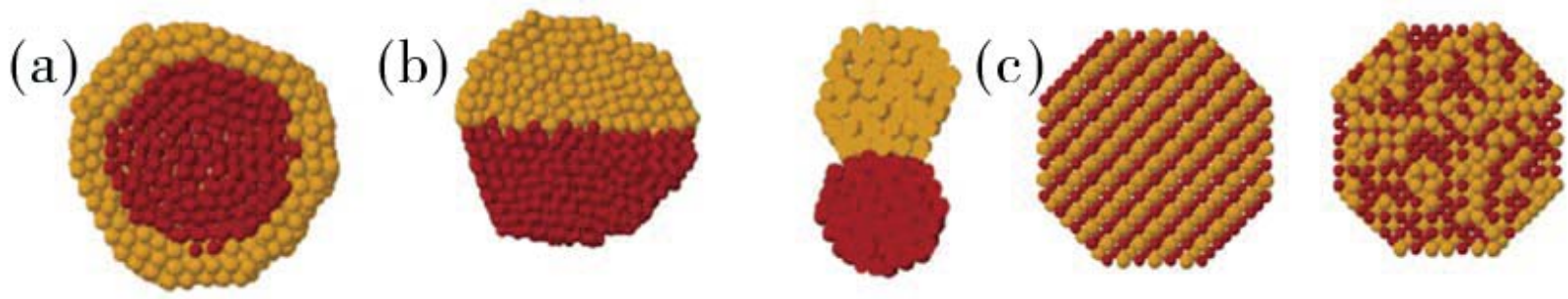

Figure 1 Schematic representation of some possible mixing patterns: core-shell (a), subcluster segregated (b), mixed (c) [16]. Reprinted with permission from Ferrando, R.; Jellinek, J.; Johnston, R. L., Chemical Reviews 2008, 108 (3), 845-910 by ACS publication. 


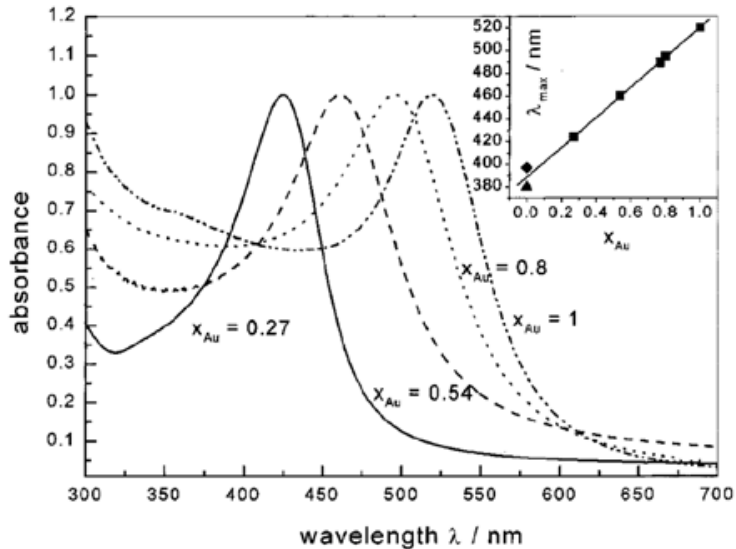

Figure 2 UV-vis absorption spectra of gold and gold-silver alloy nanoparticles with varying gold mole fractions $\mathrm{x}_{\mathrm{Au}}$. The inset shows how the absorption maximum of the plasmon band depends on the composition [44]. Reprinted with permission from Link, S.; Wang, Z. L.; El-Sayed, M. A., Journal of Physical Chemistry B 1999, 103 (18), 35293533 by ACS publication.

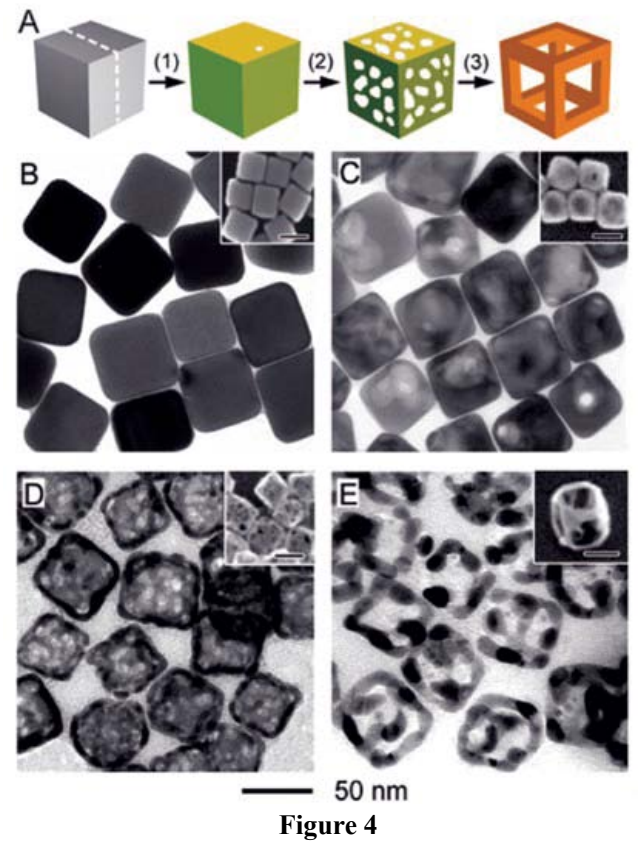

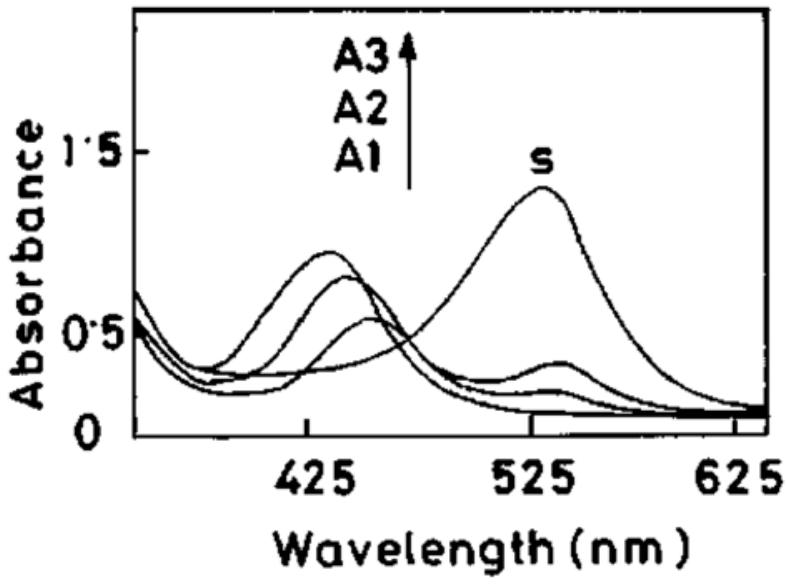

Figure 3 Initially Au particles formed by UV irradiation(s), which act as the seed particles, catalyzed the reduction of added silver ion in the presence of UV light to yield bimetallic (Aucore-Agshell) particles with different irradiate time(A1,A2,A3) [47]. Reprinted with permission from Mallik, K.; Mandal, M.; Pradhan, N.; Pal, T., Nano Letters 2001, 1 (6), 319-322 by ACS publication.
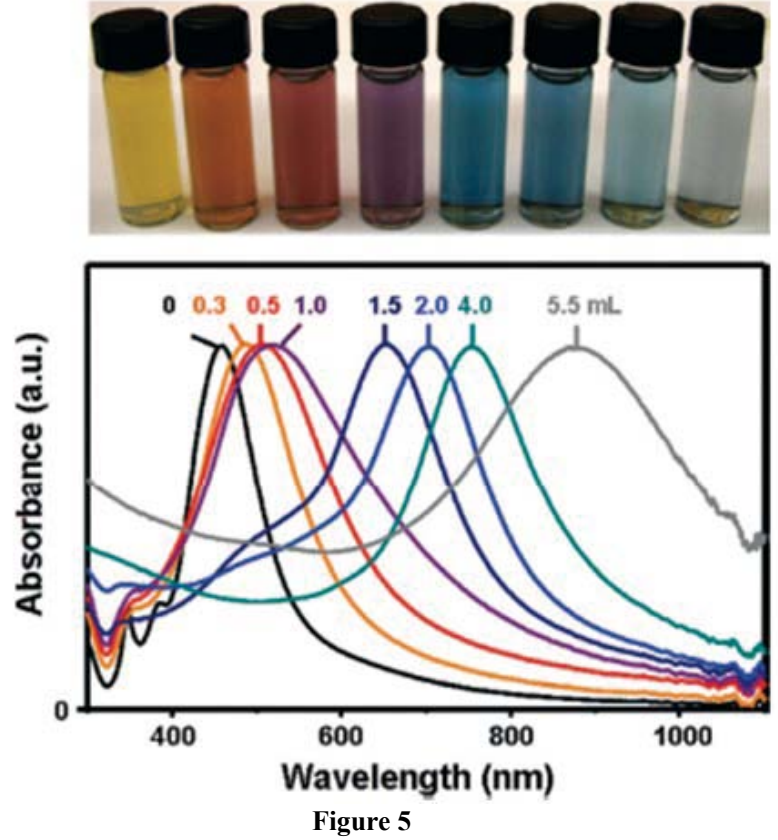

Figure 4 (A) Illustration summarizing cubic Au nanocage and Au nanoframe formation. TEM and SEM of $50 \mathrm{~nm}$ Ag nanocubes, Au/Ag nanoboxes, nanocages and nanoframes [70].

Figure 5 Top panel, vials containing Au nanocages prepared by reacting $5 \mathrm{~mL}$ of a $\sim 0.2 \mathrm{nmolL}{ }^{-1} \mathrm{Ag}$ nanocube (edge length $\approx 40 \mathrm{~nm}$ ) suspension with different volumes of a $0.1 \mathrm{mmolL}^{-1} \mathrm{HAuCl}_{4}$ solution. Lower panel, the corresponding UV-visible absorbance spectra of Ag nanocubes and Au nanocages [70]. Reprinted with permission from Skrabalak, S. E.; Chen, J. Y.; et al; Accounts of Chemical Research 2008, 41 (12), 1587-1595 by ACS publication.

during the course of the reaction. Another problem is different reduction rates of metal ions. Coreshell structure could be readily prepared through a heteronucleation process: the metal with the faster reduction forms the core and the other is the surrounding shell. For instance, AucorePtshell nanostructure usually formed when gold and platinum precursor co-reduced due to different reduction rates of gold and platinum ions [82]. To overcome these problems, Chen-Sheng Yeh and coworkers attempted to melting gold and silver nanoparticles into alloy metal particles under laser light irradiation as to the two metals are miscible in the bulk phase, owing to very similar lattice constants [80]. This method was unrestricted by solution concentrations.

In addition to gold-silver alloy nanoparticles, goldsilver alloy nanoboxes (structures with hollow interiors) and nanocages (structures with both hollow interiors and porous walls) have plasmon band in the nearinfrared region (NIR) of electromagnetic spectrum [6973]. Younan $\mathrm{Xia}$ research group synthesized nanoboxes and nanocages in large quantities using a simple galvanic replacement reaction between silver (Ag) nanocubes and chloroauric acid $\left(\mathrm{HAuCl}_{4}\right)$ in water. By controlling the titrated amount of $\mathrm{HAuCl}_{4}$ into the reaction, the LSPR peak position of gold nanocages can be precisely tuned to any wavelength of interest 


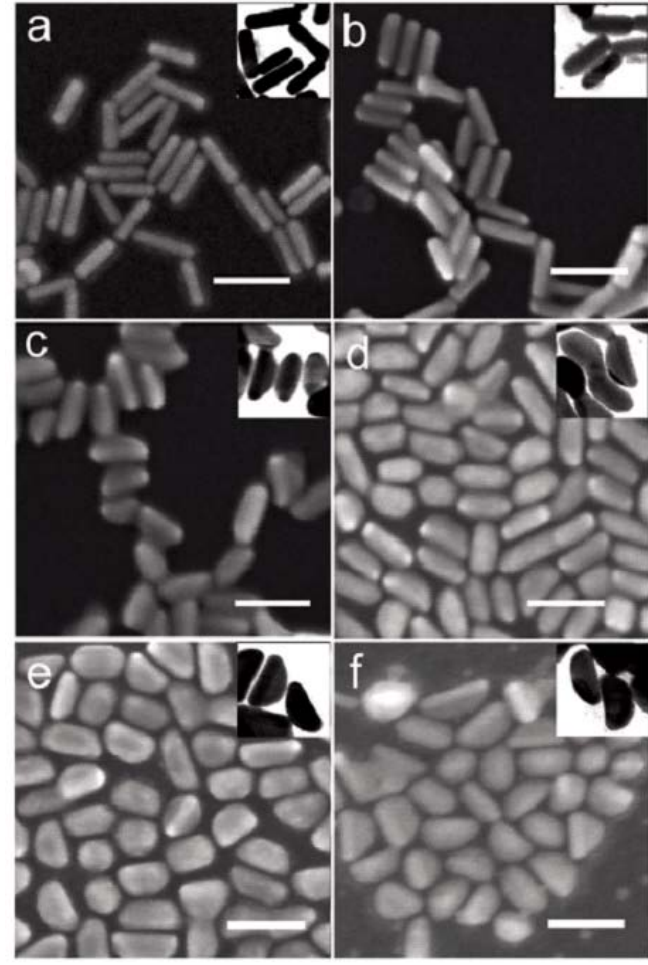

Figure 6

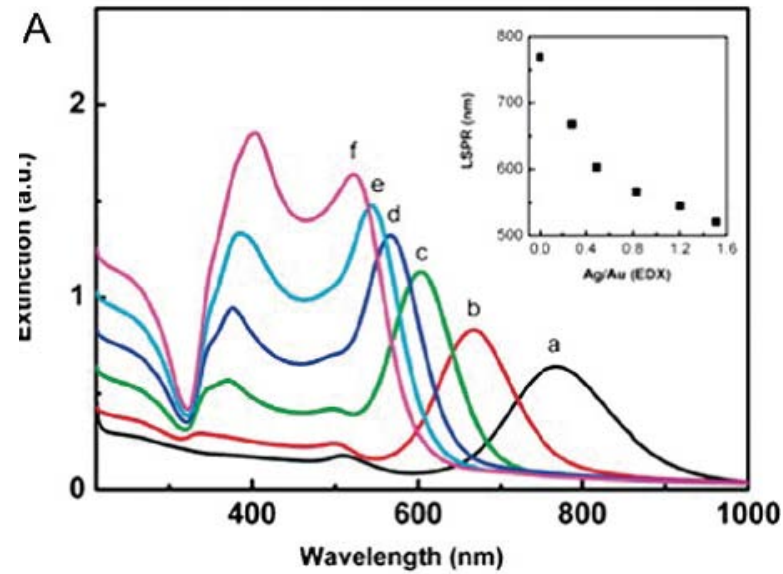

B

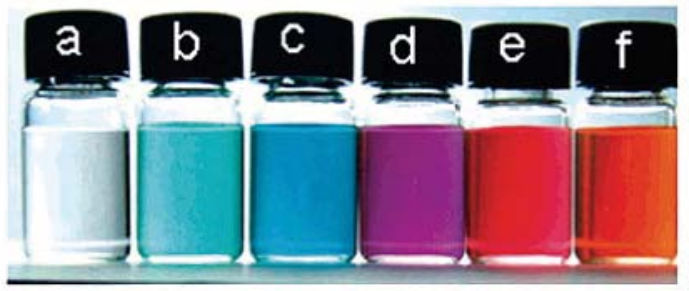

Figure 7

Figure 6 SEM images of (a) Au NRs and (b-f) Au@Ag core/shell nanocrystals with Ag/Au molar ratios of 0.28, 0.49, 0.83, 1.20, and 1.51 measured by EDX. The scale bar is $60 \mathrm{~nm}$. Insets are corresponding STEM images with the same scale bar [58].

Figure 7 (A) UV-vis extinction spectra of (a) Au NRs and (b-f) the Au@Ag core/shell nanocrystals with Ag/Au molar ratios as described in Figure 6. The inset in panel A shows the relationship between the longitudinal SPR position and the Ag/Au molar ratio. (B) Photographs of nanocrystal dispersions corresponding to the curves in panel A [58]. Reprinted with permission from Xiang, Y. J.; Wu, X. C.et al. Langmuir 2008, 24 (7), 34653470 by ACS publication.

in the range of 600-1200 $\mathrm{nm}$. Additionally, the unique hollow and porous structures of $\mathrm{Au}$ nanocages make them well suited for drug encapsulation and controlled release through the photothermal effect with NIR light. TEM and SEM images of $50 \mathrm{~nm} \mathrm{Ag} \mathrm{nanocubes,} \mathrm{Au} / \mathrm{Ag}$ nanoboxes, nanocages and nanoframes, and the UV-vis absorption spectra were shown in Figure 4 and Figure 5. Apart from gold-silver alloy nanocages, replacement reactions also used to synthesize solid gold-silver alloy nanoparticles [27]. The size and composition could be tunable by controlling the titrated amount of $\mathrm{HAuCl}_{4}$ and temperature.

The data mentioned above mainly review the synthesis methods and surface plasmon resonance (SPR) properties of nanoparticles with sphere or porous shapes. Rodlike noble nanostructures such as gold nanorods are the focus now due to the surface plasmon resonance band in near-infrared region and its application in biosensing, imagining, and photothermal therapy. Gold NRs has two surface plasmon absorption bands. One is called transverse plasmon band, relating to the absorption and scattering of light along short axis of rods, located in the visible region at ca. $520 \mathrm{~nm}$; the other is called longitudinal plasmon band, relating to the absorption and scattering of light along long axis of rods, located in the near-infrared region (NIR) of electromagnetic spectrum which is easily tunable by changing the aspect ratio of Gold NRs. Compared to gold nanorods, Ag nanorods has much narrow bandwidth (full width at half-maximum) and high absorption cross section. These advantages endow $\mathrm{Au}-\mathrm{Ag}$ alloy nanorods with multi-color imagining for high resolution [19]. The discrete dipole approximation (DDA) method had already carried out on Au-Ag alloy nanorods. It showed that the plasmon bandwidth (fwhm) is sensitive to the fraction of $\mathrm{Ag}$ in the alloy. The plasmon band becomes sharper and also increases in intensity as the fraction of $\mathrm{Ag}$ increases. A narrower bandwidth allows much easier detection of the change in the surface plasmon band maximum in response to refractive index changes in the surrounding medium. The problem here is few groups reported on their making Au-Ag alloy nanorods. Instead many groups researched on the AucoreAgshell nanorods. Xiaochun $\mathrm{Wu}$ and Sishen Xie groups prepared AucoreAgshell nanorods from homogeneous coating to anisotropic coating by gold nanorod-seeded growth [58]. Their SEM images and UVvis extinction spectra were in Figure 6 and 7. Anisotropic coating of silver resulted in an orange slice-like shape for the Au@Ag nanocrystal. The LSPR peak position of Au@Ag core/shell nanocrystals can be tuned to any wavelength in the range of 500-900 nm. Additionally, the aqueous solutions of these nanocrystals showed bright and distinctive colors when their long wavelength SPR bands were tuned across the visible spectrum. In summary, how to synthesize $\mathrm{Au}-\mathrm{Ag}$ alloy nanoparticles and nanorods in high yield and uniform size still needs our unremitting efforts. 

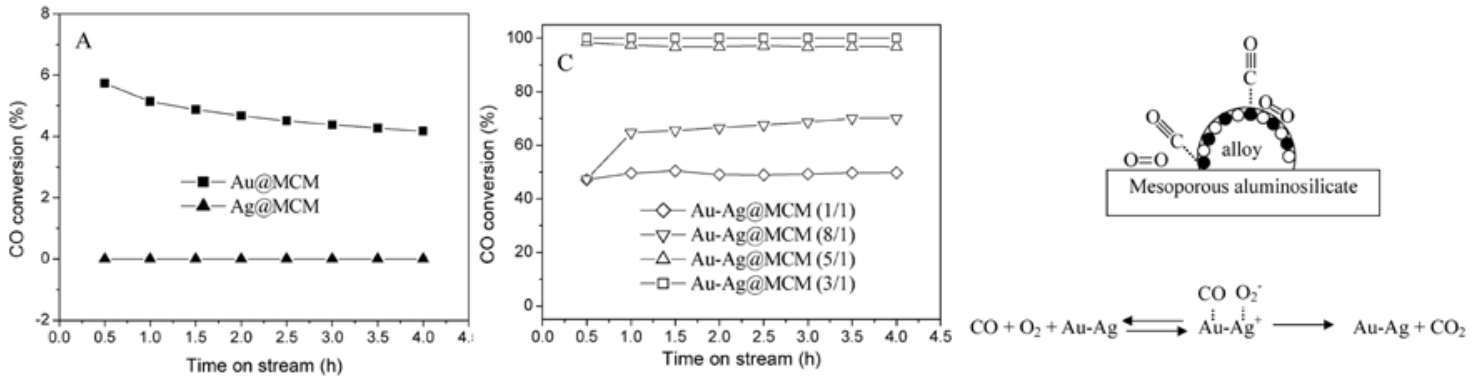

Figure $8 \mathrm{CO}$ conversion at room temperature versus time-on-stream for pure metals supported on MCM-41, CO conversion at room temperature versus time-on-stream for alloy@MCM with different Au/Ag molar ratios. The reaction gas composition is: $1 \% \mathrm{CO}, 4 \% \mathrm{O}_{2}$ and $\mathrm{He}$ as balance. A mechanism scheme for alloy@MCM catalyze process [53]. Reprinted with permission from Wang, A. Q.; Liu, J. H.; et al; Journal of Catalysis 2005, 233 (1), 186-197 by Elsevier publication.

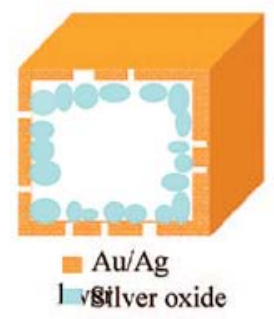

A

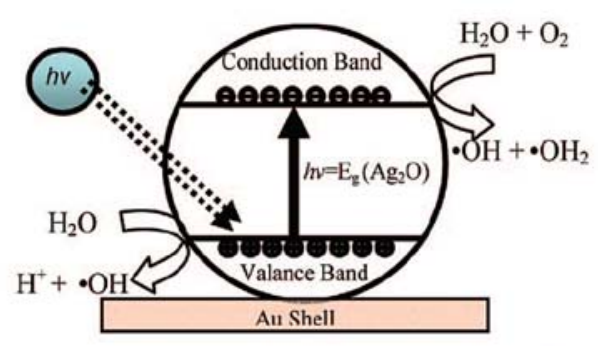

B

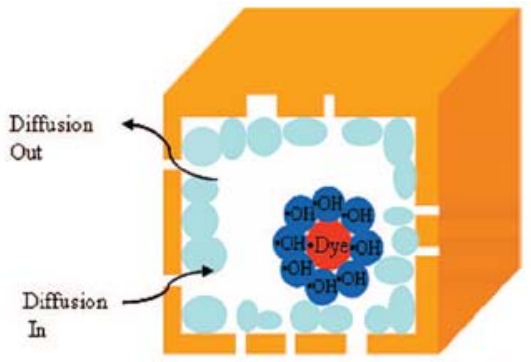

$\mathrm{C}$

Figure 9 Schematic diagrams of (A) the oxygen-treated gold nanocage, (B) the photochemical generation of hydroxyl radical by silver oxide, (C) pores in the walls allowing solution to diffuse into the cavity where photodegradation takes places. When the size of the cage reaches a certain size, it could create a cage effect that confines the hydroxyl radicals inside the cavity [84]. Reprinted with permission from Yen, C. W.; Mahmoud, M. A.; ElSayed, M. A., Journal of Physical Chemistry A 2009, 113 (16), 4340-4345 by ACS publication.

\section{Catalytic application}

There is currently much interest in the preparation and catalytic efficiency of gold catalysts in reactions such as carbon monoxide oxidation, epoxidation of propene and semi-hydrogenations. Among these catalytic application, carbon monoxide as a toxic component of air has long been targeted for removal from air. For new energy exploration, the common problem of a direct acid methanol fuel cell (DMFC) is the catalyst poisoning by formation carbon monoxide intermediate product. It has excellent application value in military, mining, and new energy. Haruta and his co-workers discovered the catalysis of gold nanoparticles in a class of oxygentransfer reactions near ambient temperature [49]. While gold nanoparticles adsorb $\mathrm{CO}$ molecules well, they do not strongly adsorb and activate oxygen molecules. The catalytic efficiency is not high enough. Silver is a poor catalyst in $\mathrm{CO}$ oxidation since at least on low index surfaces silver has low dissociation probability for $\mathrm{O}_{2}$ and $\mathrm{CO}$ desorbs too easily. But a small amount surface silver content could strongly enhance the catalytic efficiency of gold nanoparticles. Now many research groups have studied on Au-Ag alloy nanoparticles and their catalytic efficiency for oxidation of carbon monoxide. The catalytic activity has been found to be sensitive to the size of the nanoparticles, $\mathrm{Ag}$ contents, the nature of the support, and the preparation methods [50-55].

Chungyuan Mou group had made Au-Ag alloy nanoparticles supported on mesoporous aluminosilicate. $\mathrm{Au}-\mathrm{Ag}$ alloy nanoparticles were about 20-30 nm [53]
As shown in Figure 8, for pure gold nanoparticles, only under $5 \mathrm{~nm}$ diameter particles had catalytic activity for $\mathrm{CO}$ oxidation. When alloy formed, the catalytic efficiency reached about $100 \%$ although relatively large particle size. Their researches showed Au@MCM had a very low activity, whereas Ag@MCM did not exhibit any catalytic activity at room temperature. In contrast, $\mathrm{Au}-\mathrm{Ag}$ alloy catalysts showed much higher (and more stable) activity than the corresponding monometallic catalysts at low temperatures. The catalyst with an $\mathrm{Au} / \mathrm{Ag}$ ratio of 3:1 has the highest activity on which $\mathrm{CO}$ is completely converted at room temperature. A possible catalytic mechanism for Au-Ag alloy system had been proposed. While gold nanoparticles adsorb CO molecules well, they do not strongly adsorb and activate oxygen molecules. The exceptionally high activity at low temperature of Au-Ag alloy catalysts implies that oxygen can be adsorbed and activated on the alloy catalyst surface at low temperatures. The adsorption and activation of oxygen must take place on silver, and the presence of gold helps the molecular adsorption of oxygen and formation of the $\mathrm{O}_{2}^{-}$species on the silver surface. Electron transfer from silver to the antibonding orbital of oxygen molecule helps weaken its chemical bond. With a neighboring adsorbed $\mathrm{CO}$, the oxygen transfer reaction can occur easily.

Au-Ag alloy system could selective catalytic oxidize alkene to aldehyde. K. Hellgardt and coworkers studied the partial oxidation of propene over $\mathrm{Au}-\mathrm{Ag}$ alloy and monometallic Ag [83]. There were two partial oxidation products, namely acrolein and 1,5-hexadiene, in addition to carbon dioxide. Au-Ag alloy was more selective 
towards acrolein formation than monometallic Ag. This possibly promoted by oxygen adsorbed molecularly on exposed Ag surfaces whereas 1,5-hexadiene formation is promoted by oxygen species adsorbed atomically on silver surfaces.

Younan Xia group have evaluated the catalytic properties of Au-Ag nanocages, nanoboxes, and solid nanoparticles using a model reaction based on the reduction of p-nitrophenol by $\mathrm{NaBH}_{4}$ [69]. AuAg nanocages are catalytically more active than both the nanoboxes and nanoparticles probably due to their extremely thin but electrically continuous walls, and the accessibility of both inner and outer surfaces through the pores in the walls. M. A. El-Sayed group made use of Au-Ag nanocages to photodepredating methyl orange (MO) [84]. It had even more efficient than photodegradation reaction using semiconductor nanomaterials, such as $\mathrm{TiO}_{2}$ and $\mathrm{ZnO}$. They explained silver is easily oxidized to silver oxide even at $300 \mathrm{~K}$, and the formation of silver oxide $\left(\mathrm{Ag}_{2} \mathrm{O}\right)$ is extremely stable in ambient conditions. The effective catalyzer is indeed silver oxide in interior walls. They proposed that the catalytic reaction occurred inside the nanocages. The more surface area of Ag inside the walls, the more hydroxyl radicals are generated, in addition an increasing in the collision probability of radicals with methyl orange (MO). The pore size of $\mathrm{Au}-\mathrm{Ag}$ nanocages is an important condition for high catalysis. The channel (pore) sizes should sufficiently large enough to allow the reactants and products to diffuse into and out of the cavity but small enough to keep the radical steady state concentration high. The possible mechanism is showed in Figure 9.

As a consequence of the above mentioned, $\mathrm{Au}-\mathrm{Ag}$ bimetallic nanoparticles have super applicable tendency of catalysis. The issues now are how to transform these scientific results to practical application, and how to transform the laboratory synthesis to large scale industrial production. Apart from sphere shaped nanoparticles, more investigates need to prepare anisotropic gold-silver nanostructures.

\section{Surface-enhanced Raman Scattering (SERS) application}

Surface-enhanced Raman Scattering (SERS) was discovered thirty years ago. It was reported that with SERS technique the signal for target molecule could be $10^{14}-10^{15}$ higher than normal raman scattering. The advantage of this technique is single molecule detection $[85,86]$. Scientist discovered silver, copper and gold metal materials were dominant SERS substrates. In the first decade [87], scientific research workers found surface roughness, either atomic scale or nanoscale, was required for SERS. The largest enhancement occurs for surfaces which are rough on the nanoscale (10-100 $\mathrm{nm})$. But recent researches pointed out the roughness for increasing the adsorbance of target molecule could not agree with the enhanced SERS intensities. Now there are two mechanisms accepted by investigators. They are electromagnetic enhancement and chemical enhancement. The electromagnetic field of light at the surface can be greatly enhanced under conditions of surface plasmon excitation; the amplification of both the incident laser field and the scattered raman field through their interaction with the surface constitutes the electromagnetic mechanism. Chemical enhancement aims at electronic resonance and charge transfer between a molecule and a metal surface resulting in an increase in the polarizability of the molecules. In common, electromagnetic enhancement is exponential higher than chemical enhancement. Gold and silver are noble materials which have surface plasmon excitation by incident light. Therefore, researches on SERS mechanisms and making gold-silver new nanomaterials are required. There are many researches on SERS with monometallic nanoparticles, such as silver nanocubes, silver nanowires, silver nanospheres, gold nanorods, gold nanocages, and gold nanowires et al, but only a few papers on gold-silver alloy nanoparticles [43,62,68,88-92]. EM theoretical predicted the largest SERS enhancement occurred when a stronger plasmon resonance with the excitation light. The surface plasmon resonance of goldsilver alloy nanoparticles could be tuned by altering component of gold and silver. Therefore the largest SERS enhancement could be tuned.

Kwan Kim and coworkers did experiments on SERS spectra of pyridine at full coverage with pure gold, silver nanoparticles and $\mathrm{Au}_{0.7} \mathrm{Ag}_{0.3}$ alloy nanoparticles [62]. They found the SERS spectral pattern from alloy nanoparticle was closely resembles that of pure silver nanoparticle, and it substantially was different to that from the mechanical mixture of the gold and silver nanoparticle solution consisting of $70 \%$ partial gold nanoparticles and $30 \%$ partial silver nanoparticles as shown in Figure 10. They attributed the phenomenon to surface enrich of Ag atom. They indicated that although no crystal lattice mismatch of gold and silver, there was a core gold rich and shell silver rich alloy nanoparticle formation when the particle is small to dozens of nanometer. Therefore they pointed out SERS spectral could be used to distinguish different nanostructures. Erkang Wang group made silver-gold bimetallic nanostructures(a mean diameter of $100 \mathrm{~nm}$ ) with hollow interiors, and studied SERS activity using p-aminothiophenol (p-ATP) as a probe molecule [88]. The enhancement factors (EF) of silver-gold bimetallic hollow nanostructures were 30-40 times larger than that at silver nanoparticles with solid interiors. P. R. Sajanlal and T. Pradeep made bimetallic mesoflowers (MFs) [89]. First they prepared gold nanoflowers, then these MFs were used for the subsequent overgrowth of silver and other metal resulting in bimetallic MFs. Silver overgrowth was prominent at the tip compared to the center. Stronger SERS activity at the tip than that at the body of the Au-Ag MFs was observed. Raman images of AuMF and Au/AgMF were in Figure 11. For this structure, the expected electric field enhancement of spiky tips could also contribute to the high SERS activity at the tip. Here the charge transfer interaction between the ligand and the Ag-coated Au substrate in view of the difference in electro-negativity of $\mathrm{Au}$ and Ag worked also. Zhongqun Tian prepared core- 
http://nanobe.org
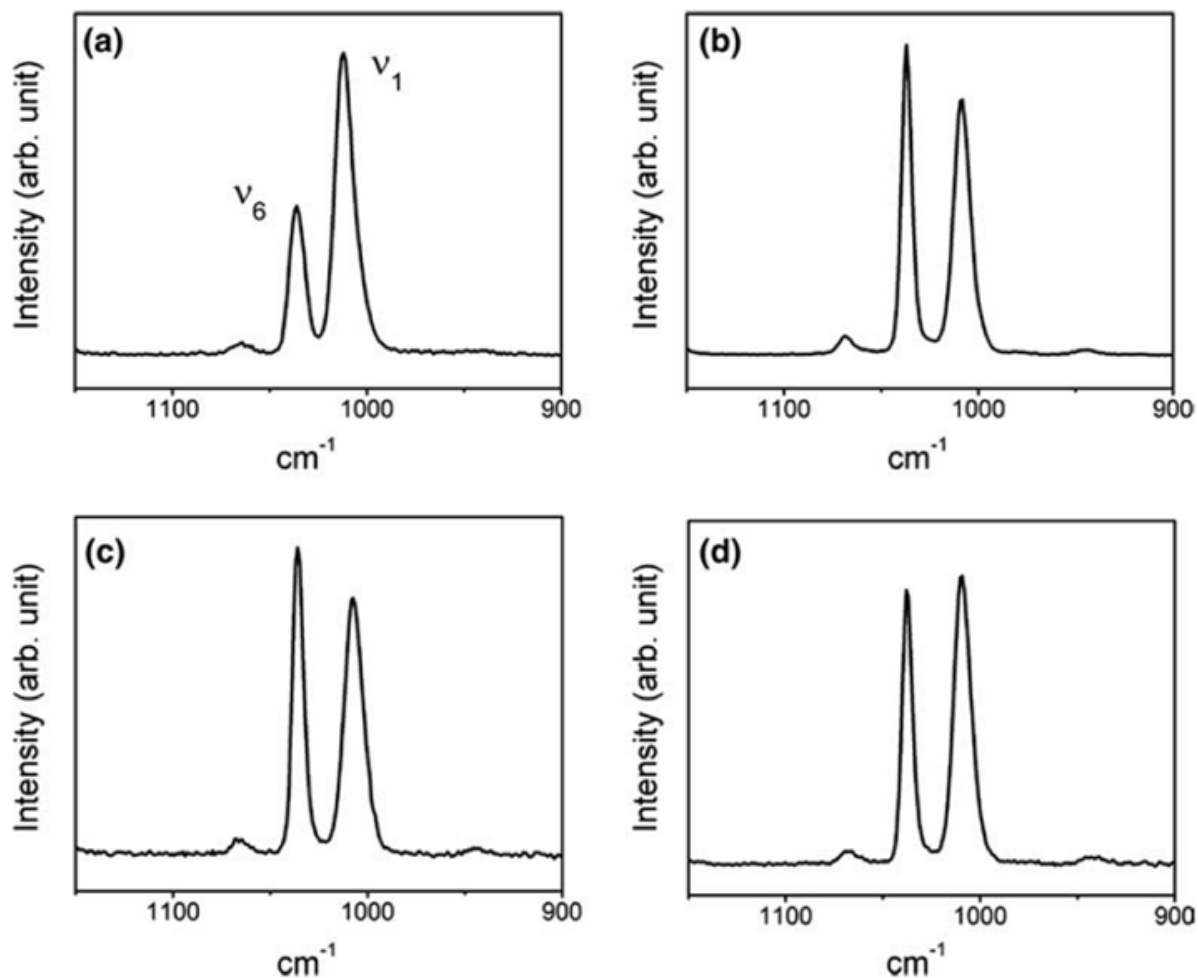

Figure 10 SERS spectra of pyridine at full coverage limit for (a) $\mathrm{Au}$, (b) $\mathrm{Ag}$, (c) Au0.7 Ag0.3 sols, taken using 632.8-nm radiation as the excitation source, (d) Similar SERS spectrum taken after adding pyridine into a 7:3 mechanical mixture of Au and Ag sols [62]. Reprinted with permission from Kim, K.; Kim, K. L.; Lee, S. J., Chemical Physics Letters 2005, 403 (1-3), 77-82 by Elsevier publication.
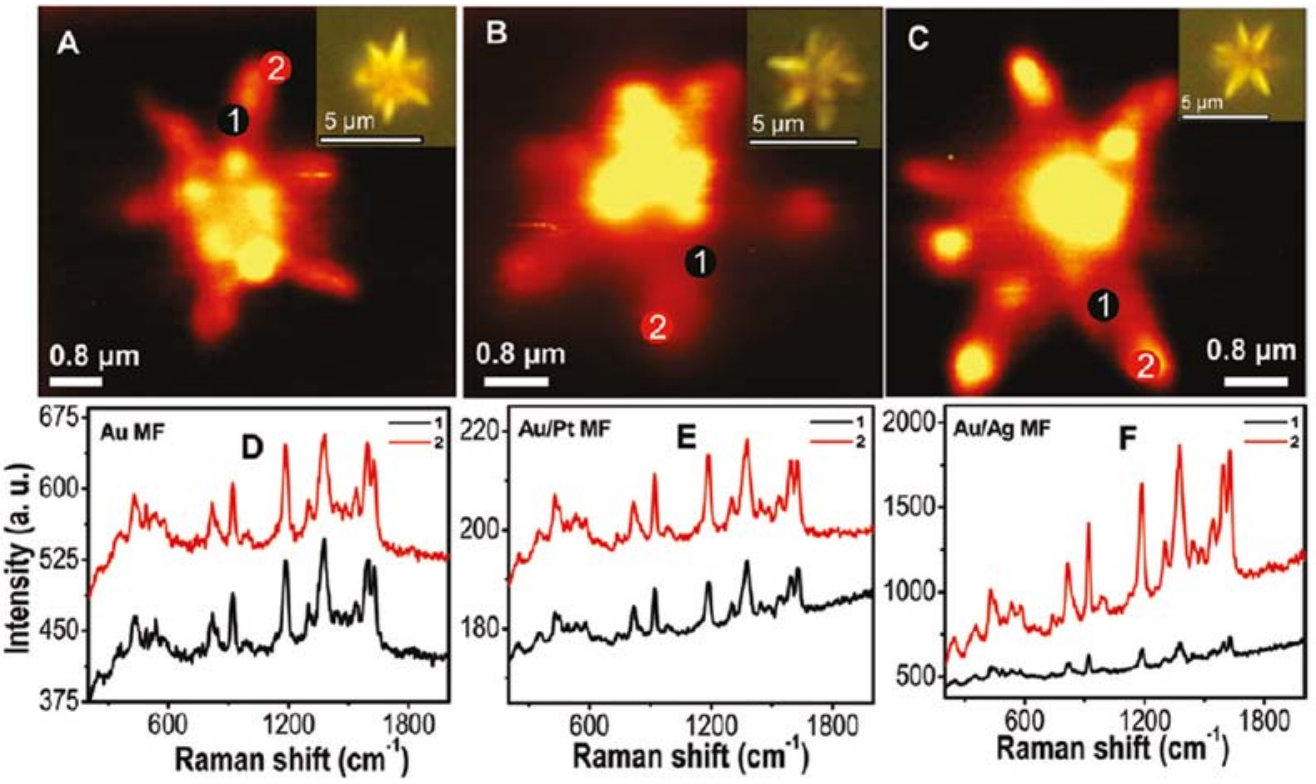

Figure 11 (A-C) Raman images of variousMFs: (A) AuMF, (B) Au/PtMF, and (C) Au/AgMF. (D), (E), and (F) are the Raman spectra collected from the points marked in the images A, B, and C, respectively [89]. Reprinted with permission from Sajanlal, P. R.; Pradeep, T., Langmuir 2010, 26 (11), 8901-8907 by ACS publication.

shell silver-gold nanoparticles (AgcoreAushell) by coating Au layers over Ag seeds by a seeded growth method [43]. The strongest SERS signals of thiophenol and p-aminothiophenol on the nanostructure surface were 10 times higher than Ag nanoparticles. They attributed this interesting result to the presence of some pinholes on the surface that act as hot spots for the electromagnetic field enhancement. Although many studies have done on goldsilver alloy nanostructures, this region even needs more powerful research. For examples, chemical enhancement is also an important effect of SERS. Some molecules have strong interaction with certain metal surface which is not good SERS substrate. How to enhance the SERS activity of inert substrate should be deep researched. May be it needs to make alloy, to make core-shell structure or some other structures. The application of SERS from gold, silver, cropper to wide range materials needs more research.

\section{Conclusions}

Bimetallic gold-silver nanoparticles have shown 
desirable optical properties, catalytic and surfaceenhanced raman scattering (SERS) properties. There are still extensive issues remained to be addressed. Now most efforts have made on sphere shape gold-silver alloy nanoparticles and core-shell nanoparticles. Considering anisotropic shaped nanoparticles are ideal building blocks for engineering and tailoring nanoscale structures for specific technological applications, for an example, gold nanorods and gold nanocages have more advantages than gold nanospheres in surface plasmon resonance and biosensor, bioimage, photothermal therapy et al. How to make gold-silver bimetal with certain shape (cube, octahedron, decahedron, nanorod, nanobar, nanobox, nanocage et al.) with good properties and detail growth principles need more efforts. How to scale up bimetallic gold-silver nanoparticles to the industrial production is urgent. Is there a phenomenon of phase segment or alloying in nanometer scale for random diffusion metal in bulk scale should study detailed. And the driving force could guide more basic scientific research. Aside from the SERS application, their utility in biological applications, such as multicolor image, biosensor will open us new valuable problems to be solved. We have long way to go in this research area to achieve the valuable application as soon as possible.

\section{Acknowledgments}

This work is supported by the National Key Basic Research Program (973 Project) (2010CB933901), National 863 Hi-tech Project (2007AA022004),Important National Science \& Technology Specific Project (2009ZX10004-311), National Natural Scientific Fund (No. 20771075 and No.20803040), Special project for nano-technology from Shanghai (No.1052nm04100), New Century Excellent Talent of Ministry of Education of China (NCET-08-0350), Shanghai Science and Technology Fund (10XD1406100).

\section{References}

1. Maier S A, Brongersma M L Kik PG, Meltzer S, Requicha A A G, Atwater H A. Plasmonics - A route to nanoscale optical devices. Adv. Mater. 2001;13:1501-1505. doi:10.1002/1521-4095 (200110)13:19<1501:AID-ADMA1501>3.0.CO;2-Z

2 .Law M, Sirbuly D J, Johnson J C, Goldberger J, Saykally R J, Yang P D.Nanoribbon waveguides for subwavelength photonics integration. Science 2004;305:1269-1273. doi:10.1126/science.1100999

3. Murphy C J, Gole A M, Hunyadi S E, Stone J W, Sisco P N, Alkilany A,Kinard B E, Hankins P., Chemical sensing and imaging with metallic nanorods. Chem. Commun. 2008;5:544-557. doi:10.1039/ b711069c

4 .Mohamed M B, Volkov V, Link S, El-Sayed M A. The 'lightning' gold nanorods: fluorescnce enhancement of over a million compared to the gold metal. Chem. Phys. Lett. 2000;317 :517-523. doi:10.1016/ S0009-2614(99)01414-1

5 .Dapeng Yang, Daxiang Cui, Advances and Prospects of Gold Nanorods. Chem.-Asian J. 2008;3:2010-2022. doi:10.1002/asia.2008 00195

6. Li C Z, Male K B, Hrapovic S, Luong J H T. Fluorescence properties of gold nanorods and their application for DNA biosensing. Chem. Commun. 2005;313924-3926.doi:10.1039/b504186d

7 .Orendorff C J, Gearheart L, Jana N R, Murphy C J. Aspect ratio dependence on surface enhanced Raman scattering using silver and gold nanorod substrates. Phys. Chem. Chem.Phys. 2006;8:165-170. doi:10.1039/b512573a

8.Zhao D, Xu B Q. Enhancement of Pt utilization in electrocatalysts by using gold nanoparticles. Angew. Chem.-Int. Edit. 2006;45:49554959.doi:10.1002/anie.200600155

9. Lee H, Habas S E, Kweskin S, Butcher D, Somorjai G A,Yang P D. Morphological control of catalytically active platinum nanocrystals.
Angew. Chem.-Int. Edit. 2006;45 :7824-7828. doi:10.1002/anie.2006 03068

10.Narayanan R, El-Sayed M A. Changing catalytic activity during colloidal platinum nanocatalysis due to shape changes: Electrontransfer reaction. J. Am. Chem. Soc. 2004;126:7194- 7195. doi:10. $1021 / \mathrm{ja} 0486061$

11.Wang A Q, Chang C M, Mou C Y. Evolution of catalytic activity of $\mathrm{Au}-\mathrm{Ag}$ bimetallic nanoparticles on mesoporous support for $\mathrm{CO}$ oxidation. J. Phys. Chem. B 2005;109:18860-18867. doi:10.1021/ jp051530q

12.Eghtedari M, Oraevsky A,Copland J A,Kotov N A,Conjusteau A, Conjusteau A, Motamedi M. High sensitivity ofsensitivity of in vivo detection of gold nanorods using a laser optoacoustic imaging system. Nano Lett. 2007;7:1914-1918.doi:10.1021/n1070557d

13. Wiley B J, Wang Z H, Wei J, Yin Y D,Cobden D H, Xia Y N. Synthesis and electrical characterization of silver nanobeams. Nano Lett. 2006;6:2273-2278 doi:10.1021/n1061705n

14.Loo C, Lowery A, Halas N, West J, Drezek R. Immunotargeted nanoshells for integrated cancer imaging and therapy. Nano Lett. 2005; 5:709-711.doi:10.1021/n1050127s

15.Eustis S, El-Sayed M A. Why goldnanoparticles are more precious than pretty gold:Noble metal surface plasmon resonance and its enhancement of the radiative and nonradiative properties of nanocrystals of different shapes. Chem. Soc. Rev. 2006;35:209217 doi:10.1039/b514191e

16.Ferrando R, Jellinek J, Johnston R L. Nanoalloys: From theory to applications of alloy clusters and nanoparticles. Chem. Rev. 2008; 108:845-910.doi:10.1021/cr040090g

17.Herricks T, Chen J Y, Xia Y N. Polyol synthesis of platinum nanoparticles: Control of morphology with sodium nitrate. Nano Lett. 2004;4:2367-2371. doi:10.1021/n1048570a

18.Lee K S, El-Sayed M A. Dependence of the enhanced optical scattering efficiency relative to that of absorption for gold metal anorods on aspect ratio, size, end-cap shape, and medium refractive index. J. Phys. Chem. B 2005;109:20331-20338. doi:10.1021/jp0543 $85 \mathrm{p}$

19.Lee K S, El-Sayed M A. Gold and silver nanoparticles in sensing and imaging:Sensitivity of plasmon response to size, shape, andmetal composition. J. Phys. Chem. B 2006 ; 110: 19220-19225. doi:10.102 $1 /$ jp06253 6y

20.Link S, Mohamed M B, El-Sayed M A. Simulation of the optical absorption spectra of gold nanorods as a function of their aspect ratio and the effect of the medium dielectric constant. J. Phys. Chem. B. 1999;103:3073-3077.doi:10.1021/jp990183f

21.Liz-Marzan L M. Tailoring surface plasmons through the morphology and assembly of metal nanoparticles. Langmuir. 2006; 2:32-41.doi: $10.1021 / 1 \mathrm{a} 0513353$

22.Sherry L J,Chang S H,Schatz G C,Van Duyne R P, Wiley B J, Xia Y $\mathrm{N}$. Localized surface plasmon resonance spectroscopy of single silver nanocubes. Nano Lett.2005; 5:2034-2038. doi:10.1021/n10515753

23.Tao A, Sinsermsuksakul P, Yang P. Tunable plasmonic lattices of silver nanocrystals. Nat. Nanotechnol. 2007;2:435-440. doi:10.1038/ nnano.2007.189

24.Wiley B, Sun Y G, Mayers B, Xia Y N. Shape-controlled synthesis of metal nanostructures: The case of silver. Chem.-Eur. J. 2005;11: 454-463.doi:10.1002/chem. 200400927

25.Xiong Y J, Wiley B, Chen J Y, Li Z Y, Yin Y D, Xia Y N. Corrosionbased synthesis of single-crystalPd nanoboxes and nanocagesand their surface plasmon properties. Angew. Chem.-Int. Edit. 2005;44: 7913-7917.doi:10.1002/anie.200502722

26.Sun Y G, Xia Y A. Alloying and dealloying processes involved in the preparation of metal nanoshells through a galvanic replacement reaction. Nano Lett. 2003;3:1569-1572. doi:10.1021/n1034765r

27 .Zhang Q B, Lee J Y, Yang J, Boothroyd C, Zhang J X. Size and composition tunable Ag-Au alloy nanoparticles by replacement reactions. Nanotechnology. 2007;18:245605.doi:10.1088/0957-448 $4 / 18 / 24 / 245605$

28.Lu X M, Au L, McLellan J, Li Z Y, Marquez M, Xia Y N, Fabrication of cubic nanocages and nanoframes by dealloying $\mathrm{Au} / \mathrm{Ag}$ alloy nanoboxeswith an aqueous etchant based on $\mathrm{Fe}\left(\mathrm{NO}_{3}\right)_{3}$ or $\mathrm{NH}_{4} \mathrm{OH}$. Nano Lett. 2007; 7:1764-1769. doi:10.1021/n10708381

29. Chen J Y, Wiley B, McLellan J, Xiong Y J, Li Z Y, Xia Y N. Optical properties of Pd-Ag and Pt-Ag nanoboxes synthesized via galvanic replacement reactions. Nano Lett. 2005;5: 2058-2062. doi:10.1021/ nl051652u

30 .Chandran S P, Ghatak J, Satyam P V, Sastry M. Interfacial deposition 
of Ag on Au seeds leading to AucoreAgshell in organic media. $J$ Coll. Inter. Sci. 2007;312 : 498-505. doi:10.1016/j.jcis.2007.03.032

31.Yang J, Lee J Y, Too H P. Core-shell Ag-Au nanoparticles from replacement reaction in organic medium. J. Phys. Chem. B 2005; 109:19208-19212.doi:10.1021/jp052242x

32.Henglein A. Reduction of $\operatorname{Ag}(\mathrm{CN})(2)(-)$ on silverand platinum colloidal nanoparticles. Langmuir. 2001;17:2329-2333. doi:10.1021/ la001081f

33.Lu L H, Wang H S, Xi S Q, Zhang H J. Improved size control of large palladium nanoparticles by a seeding growth method. J. Mater. Chem. 2002;12:156-158.doi:10.1039/b109797k

34.Watanabe K, Menzel D, Nilius N, Freund H J. Photochemistry on metal nanoparticles. Chem. Rev. 2006;106:4301-4320. doi:10.1021/ cr050167g

35.Daniel M C, Astruc D. Gold nanoparticles: Assembly, supramolecular chemistry, quantum- size-related properties, and applicationstoward biology,catalysis, nanotechnology. Chem. Rev. 2004; 104:293-346. doi:10.1021/cr030698+

36.Bonnemann H, Richards R M. Nanoscopic metal particles-Synthetic methods and potential applications. Eur. J. Inorg. Chem. 2001; 10:2455-2480.doi:10.1002/1099-0682(200109)2001:10<2455::AIDEJIC2455>3.0.CO;2-Z

37.Fan F R, Liu D Y, Wu Y F, Duan S, Xie Z X, Jiang Z Y, Tian Z Q. Epitaxial growth of heterogeneous metal nanocrystals: From gold nano-octahedra to palladium and silver nanocubes. J. Am. Chem. Soc. 2008;130:6949-6950.doi:10.1021/ja801566d

38. Toshima N, Yonezawa T. Bimetallic nanoparticles-novel materials for chemical and physical applications. New J. Chem. 1998;22:11791201 doi: $10.1039 / \mathrm{a} 805753 \mathrm{~b}$

39.Mulvaney P. Surface plasmon spectroscopy of nanosized metal particles. Langmuir 1996;12:788-800. doi:10.1021/la9502711

40.Alayoglu S, Nilekar A U, Mavrikakis M, Eichhorn B. Ru-Pt coreshell nanoparticles for preferential oxidation of carbon monoxide in hydrogen. Nat. Mater. 2008;7:333-338. doi:10.1038/nmat2156

41.Toshima N, Shiraishi Y, Shiotsuki A, Ikenaga D, Wang Y. Novel synthesis, structure and catalysis of inverted core/shell structured Pd/Pt bimetallic nanoclusters. Eur. Phys. J. D.2001;16:209-212. doi:10.1007/s100530170094

42.Hu J W, Li J F, Ren B, Wu D Y, Sun S G, Tian Z Q. Palladiumcoated gold nanoparticles with a controlled shell thickness used as surface-enhanced Raman scattering substrate. J. Phys. Chem. C 2007;111:1105-1112.doi:10.1021/jp0652906

43.Cui Y, Ren B, Yao J L, Gu R A, Tian Z Q.Synthesis of Agcore Aushell bimetallic nanoparticles for immunoassay based on surfaceenhanced Raman spectroscopy. J. Phys. Chem. B 2006;110:40024006. doi:10.1021/jp056203x

44.Link S, Wang Z L, El-Sayed M A. Alloy formation of gold-silver nanoparticles and the dependence of the plasmon absorption on their composition. J. Phys. Chem. B 1999;103:3529-3533. doi:10.1021/ jp990387w

45.Mallin M P, Murphy C J. Solution-phase synthesis of sub-10 nm AuAg alloy nanoparticles. Nano Lett. 2002;2:1235-1237. doi:10.1021/ n1025774n

46.Pal A, Shah S, Devi S. Synthesis of Au, Ag and Au-Ag alloy nanoparticles in aqueous polymer solution. Coll. Surf. A-Phys. Eng. Asp. 2007;302: 51-57.

47.Mallik K, Mandal M, Pradhan N, Pal T. Seed mediated formation of bimetallic nanoparticles by UV irradiation: A photochemical approach for the preparation of "core-shell" stype tructures. Nano Lett. 2001;1:319-322.doi:10.1021/n10100264

48.Luis M, Liz-Marzan A P P. Stable Hydrosols of Metallic and Bimetallic Nanoparticles Immobilized on Imogolite Fibers. J. Phys. Chem. 1995;99:15120-15128.doi:10.1021/j100041a031

49 .Haruta A. When gold is not noble: Catalysis by nanoparticles. Chem. Rec. 2003; 3 :75-87. doi: $10.1002 /$ tcr. 10053

50.Hakkinen H, Abbet W, Sanchez A, Heiz U, Landman U.Structural, electronic, and impurity-doping effects in nanoscale chemistry: Supported gold nanoclusters. Angew. Chem.-Int. Edit. 2003;42:12971300.doi:10.1002/ anie.200390334

51.Iizuka Y, Kawamoto A, Akita K, Date M, Tsubota S, Okumura M, Haruta M. Effect of impurity and pretreatment conditions on the catalytic activity of Au powder for CO oxidation. Catal. Lett. 2004; 97:203-208. doi:10.1023/B:CATL.0000038585.12878.9a

52.Liu J H, Wang A Q, Chi Y S, Lin H P, Mou C Y. Synergistic effect in an Au-Ag alloy nanocatalyst: CO oxidation. J. Phys. Chem. B 2005; 109:40-43.doi:10.1021/jp044938g
53.Wang A Q, Liu J H, Lin S D, Lin T S, Mou C Y. A novel efficient Au$\mathrm{Ag}$ alloy catalyst system: preparation, activity, and characterization. J. Catal. 2005;233:186-197. doi:10.1016/j.jcat.2005.04.028

54.Chang C M, Cheng C, Wei C M. CO oxidation on unsupported Au55, Ag-55, and Au25Ag30 nanoclusters. J. Chem. Phys. 2008;128: 124710.doi:10.1063/1.2841364

55Wang A Q, Hsieh Y, Chen Y F, Mou C Y. Au-Ag alloy nanoparticle as catalyst for $\mathrm{CO}$ oxidation: Effect of $\mathrm{Si} / \mathrm{Al}$ ratio of mesoporous support. J. Catal. 2006;237:197-206. doi:10.1016/j.jcat.2005.10.030

56.Tsuji M, Miyamae N, Lim S, Kimura K, Zhang X, Hikino S Nishio M. Crystal structures and growth mechanisms of Au@Ag core-shell nanoparticles prepared by the microwave-polyol method. Cryst. Growth Des. 2006;6:1801-1807.doi:10.1021/cg060103e

57 .Mandal M, Jana N R, Kundu S, Ghosh S K, Panigrahi M, Pal T. Synthesis of Au-core-Ag-shell type bimetallic nanoparticles for single molecule detection in solution by SERS method. J. Nanopart. Res. 2004;6:53-61.doi:10.1023/ B:NANO.0000023227.17871.0f

58.Xiang Y J, Wu X C, Liu D F, Li Z Y, Chu W G, Feng L L, Zhang K, Zhou W Y, Xie S S. Gold nanorod-seeded growth of silver nanostructures:From homogeneous coating to anisotropic coating. Langmuir. 2008;24:3465-3470. doi:10.1021/la702999c

59.Park K, Vaia R A,Synthesis of Complex Au/ Ag Nanorods by Controlled Overgrowth. Adv. Mater. 2008;20:3882-3887.doi:10.1002/ adma.200800613

60.Huang C C, Yang Z S, Chang H T,Synthesis of dumbbell-shaped $\mathrm{Au}-\mathrm{Ag}$ core-shell nanorods by seed-mediated growth under alkaline conditions. Langmuir:2004;20:6089-6092.doi:10.1021/la048791w

61.Liu M Z,Guyot-Sionnest P, Synthesis and optical characterization of $\mathrm{Au} / \mathrm{Ag}$ core/shell nanorods. J. Phys. Chem. B 2004;108:58825888. doi:10.1021/jp037644o

62.Kim K, Kim K, Lee S J .Surface enrichment of Ag atoms in Au/Ag alloy nanoparticles revealed by surface enhanced Raman scattering spectroscopy. Chem. Phys. Lett. 2005;403:77-82. doi:10.1016/j.cplet t.2004.12.025

63.Peng Z Q, Spliethoff B, Tesche B, Walther T, Kleinermanns K. Laser-assisted synthesis of Au-Ag alloy nanoparticles insolution. $J$. Phys. Chem. B 2006;110:2549-2554.doi:10.1021/jp056677w

64.Raveendran P, Fu J, Wallen S L. A simple and "green" method for the synthesis of Au, Ag, and Au-Ag alloy nanoparticles. Green Chem. 2006;8:34-38. doi:10.1039/b512540e

65.Wang W X, Huang Y P, Chen Q F, Xu S K, Yang D Z. Synthesis and absorption spectra properties of Au-Ag alloy nanoparticles using gallic acid as reductant. Spectrosc. Spectral Anal. 2008;28:17261729.

66.Shin Y, Bae I T, Arey B W, Exarhos G J. Facile stabilization of goldsilver alloy nanoparticles on cellulose nanocrystal. J. Phys. Chem. C 2008;112: 4844-4848. doi:10.1021/jp710767w

67.Chen D H, Chen C J. Formation and characterization of Au-Ag bimetallic nanoparticles in water-in-oil microemulsions. J. Mater. Chem. 2002;12:1557-1562.doi:10.1039/b110749f

68.Kim K, Kim K L, Choi J Y, Lee H B, Shin K S, Surface Enrichment of Ag Atoms in Au/Ag Alloy Nanoparticles Revealed by SurfaceEnhanced Raman Scattering of 2,6-Dimethylphenyl Isocyanide. $J$. Phys. Chem. C 2010;114: 3448-3453. doi:10.1021/jp9112624

69.Zeng J, Zhang Q, Chen J Y, Xia Y N. A Comparison Study of the Catalytic Properties of Au-Based Nanocages, Nanoboxes, and Nanoparticles. Nano Lett. 2010;10:30-35. doi:10.1021/n1903062e

70.Skrabalak S E, Chen J Y, Sun Y G, Lu X M, Au L, Cobley C M, Xia Y N. Gold Nanocages: Synthesis, Properties, and Applications. Acc. Chem. Res. 2008;41:1587-1595.doi:10.1021/ar800018v

71.Chen J Y, Glaus C, Laforest R, Zhang Q, Yang M X, Gidding M, Welch M J, Xia Y N. Gold Nanocages as Photothermal Transducers for Cancer Treatment. Small. 2010;6:811-817. doi:10.1002/small.200 902216

72.Au L, Zhang Q, Cobley C M, Gidding M, Schwartz A G, Chen J Y, Xia Y N. Quantifying the Cellular Uptake of Antibody-Conjugated $\mathrm{Au}$ Nanocages by Two-Photon Microscopy and Inductively Coupled Plasma Mass Spectrometry. Acs Nano 2010;4:35-42.dio:10.1021/nn9 $01392 \mathrm{~m}$

73. Yavuz M S, Cheng Y Y, Chen J Y, Cobley C M, Zhang Q, Rycenga M, Xie J W, Kim C, Song K H, Schwartz A G,Wang L H V, Xia Y N. Gold nanocages covered by smart polymers for controlled release with near-infrared light. Nat. Mater. 2009;8:935-939. doi:10.1038/ nmat 2564

74.Sanchez-Ramirez J F, Pal U, Nolasco-Hernandez L,Mendoza-Alvarez J, Pescador-Rojas J A.Synthesis and Optical Properties of Au-Ag 
Alloy Nanoclusters with Controlled Composition. J. Nanomater. 2008;620412.

75.Kariuki N N, Luo J, Maye M M, Hassan S A, Menard T, Naslund H R, Lin Y H, Wang C M, Engelhard M H, Composition- controlled synthesis of bimetallic gold-silver nanoparticles. Langmuir. 2004;20: 11240-11246. doi:10.1021/la048438q

76.Lizmarzan L M, Philipse A P.Stable Hydrosols of Metallic and Bimetallic Nanoparticles Immobilized on Imogolite Fibers. J. Chem. Phys. 1995;99:15120-15128.doi:10.1021/j100041a031

77.Han S W, Kim Y, Kim K, Dodecanethiol-derivatized Au/Ag bimetallic nanoparticles: TEM, UV/VIS, XPS, and FTIR analysis. $J$. Coll. Int. Sci. 1998;208: 272-278.doi:10.1006/jcis.1998.5812

78.Devarajan S, Vimalan B ,Sampath S. Phase transfer of Au-Ag alloy nanoparticles from aqueous medium to an organic solvent:effect of aging of surfactant on the formation of Ag-rich alloy compositions. $J$. Coll. Int. Sci. 2004;278:126-132. doi:10.1016/j.jcis.2004.05.038

79.Pal A, Shah S, Devi S. Preparation of silver-gold alloy nanoparticles at higher concentration using sodium dodecyl sulfate. Aust. J. Chem 2008;61:66-71.doi:10.1071/CH07165

80.Chen Y H, Yeh C S, A new approach for the formation of alloy nanoparticles: laser synthesis of gold-silver alloy from gold-silver colloidal mixtures. Chem. Commun. 2001;4:371-372. doi:10.1039/ b009854j

81.Lee I, Han S W, Kim K. Production of Au-Ag alloy nanoparticles by laser ablation of bulk alloys. Chem. Commun. 2001;18:1782-1783. doi:10.1039/b009854j

82.He W W, Wu X C, Liu J B, Zhang K, Chu W G, Feng L L, Hu X A, Zhou W Y, Xie S S. Pt-Guided Formation of Pt-Ag Alloy Nanoislands on Au Nanorods and Improved Methanol ElectroOxidation. J. Phys. Chem. C 2009;113:10505-10510. dio:10.1021/ jp9027707

83.Zemichael F W, Al-Musa A, Cumming I W, Hellgardt K. Propene partial oxidation over Au-Ag Alloy and Ag catalysts using electrochemical oxygen. Solid State Ionics. 2008;179:1401- 1404.

84.Yen C W, Mahmoud M A, El-Sayed M A,Photocatalysis in Gold Nanocage Nanoreactors. J. Phys. Chem. A 2009;113:4340-4345. doi:10.1021/jp811014u

85.Nie S M, Emery S R. Probing single molecules and single nanoparticles by surface-enhanced Raman scattering. Science 1997; 275:1102-1106:1102-1106. doi:10.1126/science.275.5303.1102

86. Michaels A M, Nirmal M, Brus L E. Surface enhanced Raman spectroscopy of individual rhodamine $6 \mathrm{G}$ molecules on large Agnanocrystals. J. Am. Chem. Soc. 999;121:9932-9939. doi:10.1021/ ja992128q

87.Campion A, Kambhampati P. Surface-enhanced Raman scattering. Chem. Soc. Rev. 1998;27:241-250. doi:10.1039/a827241z

88.Sajanlal P R, Pradeep T. Bimetallic Mesoflowers: Region-Specific Overgrowth andSubstrate Dependent Surface-Enhanced Raman Scattering at Single Particle Level. Langmuir. 2010;26:8901-8907. doi:10.1021/1a904676u

89. Wang Y L, Chen H J, Dong S J, Wang E K.Surface-enhanced Raman scattering of silver-gold bimetallic bimetallic nanostructures with hollow interiors. J. Chem. Phys. 2006;125:044710.doi:10.1063/1.22 16694

90.Gellner M, Kustner B, Schlucker S. Optical properties and SERS efficiency of tunable gold/silver nanoshells. Vib. Spectrosc. 2009; 50:43-47.doi:10.1016/j.vibspec.2008.07.011

91.Srnova-Sloufova I, Vlckova B, Bastl Z, Hasslett T L. Bimetallic (Ag)Au nanoparticles prepared by the seed growth method:Twodimensional assembling, characterization by energy dispersive $\mathrm{X}$-ray analysis, X-ray photoelectron spectroscopy, and surface enhanced Raman spectroscopy, and proposed mechanism of growth. Langmuir 2004; 20:3407-3415.doi:10.1021/la0302605

92.Olson T Y, Schwartzberg A M, Orme C A, Talley C E, O'Connell B, Zhang J Z. Hollow gold-silver double-shell nanospheres: Structure, optical absorption, and surface-enhanced Raman scattering. J. Phys. Chem. C 2008;112:6319-6329.doi:10.1021/jp7116714

Received 15 November, 2010; accepted 6 December, 2010; published online 16 December, 2010.

Copyright:(c) 2010 L. Feng, et al. This is an open-access article distributed under the terms of the Creative Commons Attribution License, which permits unrestricted use, distribution, and reproduction in any medium, provided the original author and source are credited. 